\title{
The Quantification of Small Visual Colour Differences
}

\section{Document Version}

Accepted author manuscript

Link to publication record in Manchester Research Explorer

\section{Citation for published version (APA):}

Hayden, S. L., \& Oulton, D. (1994). The Quantification of Small Visual Colour Differences. Society of Dyers and Colourists. Journal , 110(3), 104-111.

\section{Published in:}

Society of Dyers and Colourists. Journal

\section{Citing this paper}

Please note that where the full-text provided on Manchester Research Explorer is the Author Accepted Manuscript or Proof version this may differ from the final Published version. If citing, it is advised that you check and use the publisher's definitive version.

\section{General rights}

Copyright and moral rights for the publications made accessible in the Research Explorer are retained by the authors and/or other copyright owners and it is a condition of accessing publications that users recognise and abide by the legal requirements associated with these rights.

\section{Takedown policy}

If you believe that this document breaches copyright please refer to the University of Manchester's Takedown Procedures [http://man.ac.uk/04Y6Bo] or contact uml.scholarlycommunications@manchester.ac.uk providing relevant details, so we can investigate your claim.

\section{OPEN ACCESS}




\title{
The quantification of small visual colour differences
}

\author{
Sarah L Hayden and David P Oulton
}

\author{
Dept of Textiles, UMIIST, PO Box 88, Manchester M60 1QD, UK
}

The Shademaster system, developed at UMIST, was used to explore the use of on-screen colour in order to assess the reliability of the $\mathrm{CMC}(I ; c)$ formula as a measurement of small colour differences. Differences limited to almost pure hue, chroma and lightness changes around nine colour centres were judged by a group of ten observers. Each colour difference pair was presented on-screen in the form of a divided tile. The colour difference between each pair was measured using a Bentham telespectroradiometer and by a method using reverse transformation of RGB to CIE coordinates. The resultant measurements were used to determine the visibility of a particular colour difference, how that visibility varied from colour to colour and how it varied in different directions (i.e. hue, chroma or lightness). The data obtained from the judgements were used to construct perceptibility boundaries around each colour centre.

\section{INTRODUCTION}

Visibility of small colour differences

In all areas of design, science and industry there is a need for identifying and measuring, by means of numerical specification, the appearance of a colour to the average man or woman. Various methods have been developed to frocluce ar: accurate and comprehensible method of colour measurement.

The evaluation of colour differences is known to be quite a compiex subject with many variables affecting it and, over the years, several different formulae have been devised to enable the calculation of colour differences. In this study the performance of just one of those equations, the CMC $(l: c)$ formula, is examined as a measure of justvisible colour differences. The Shademaster CAD system (Figure 1), developed at UMIST, is able to display on screen an unlimited number of colour-difference pairs and is therefore a convenient method of observing a colour difference at its threshold of visibility. Shademaster has been used to determine how the visibility of small colour differences varies from colour to colour by examining nine different colour centres and, as colour is threedimensional, how this visibility varies in each of the three directions of hue, metric chroma and lightness. The paper is firstly concerned with the validity of on-screen assessment of small colour differences. It also shows that it is possible to establish the position of the boundaries of visibility about each colour centre.

Development of the $\mathrm{CMC}(l: c)$ colour-difference formula Provided colour space is Euctidian, the distance between two points represents the difference between the two

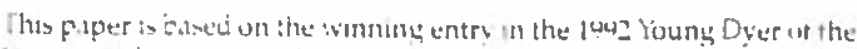

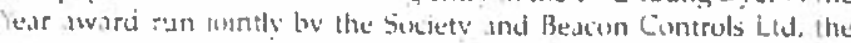

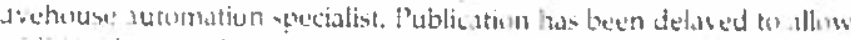

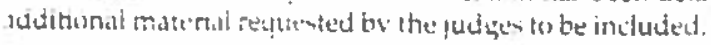

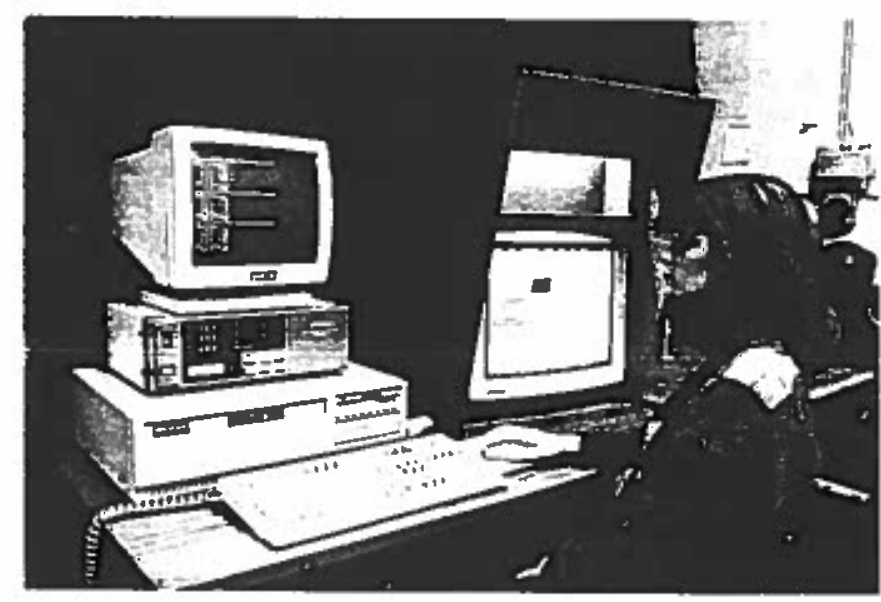

Figure 1 The Shademaster system

colours and can be calculated using Pythagoras's theorem. This distance is given the symbol $\Delta E, \Delta$ (the Greek letter delta) signifying 'difference in', and $E$ (the first letter of the German word Empfindung) meaning sensation. However, as CIE XYZ colour space is non-uniform, the relationship between the two points in colour space is not linear. Consequently further investigation led to the development of $L a b$, colour space which is more uniform.

In 1970 the Colour Measurement Committee (CMC) of the Society of Dyers and Colourists recommended the Adams-Nickerson (ANLAB) colour-difference formula for general use in the UK [1]. In 1976 the CIE (Commission Internationale de l'Éclairage or International Commission on Illumination) simplified this equation to establish the CIELAB colour space and colour-difference formula [2]. However, both the ANL.AB and CIELAB systems were seen to display a lack of uniformity, and subsequently many attempts were made to produce an improved coluurdifterence formula. Investigations by the CMC resulted in the development of the C.VC $(l: c)$ colourdifference formula [3], which was published as a British Standard (BS 6923) in September 1988 (Eyn 1) [t]: 


$$
\Delta E=\frac{\Delta L}{l S_{L}}+\frac{\Delta C}{c S_{C}}-\frac{\Delta H}{S_{H}}
$$

where $J E$ is the colour difference

$\Delta I$ is the lightness difference

$\Delta C$ is the chroma difference

$\Delta H$ is the hue difference

$S_{L} S_{C}$ and $S_{H}$ are the lengths of the half-axes of the ellipsoid defining unit $\Delta E$

$I$ and $c$ are relative tolerances and can be varied depending on the application for which the formula is required.

For perceptibility data, the equation is used as $C M C(1: 1)$, whereas for acceptability data it is used as CMC(2:1). This formula has been shown to give better agreement with experimental results than any other published formula $[3,9]$. It has now been established as the recommended method for colour-difference calculations for quality control and colour management in industry.

\section{Tolerance ellipses}

The results from colour-difference experiments can be conveniently compared and contrasted by constructing a set of tolerance ellipses, or ellipsoids, about each individual colour centre under analysis. These ellipses were first developed by MacAdam following investigations involving over 25000 colour matching observations [5]. Around each chromaticity centre studied, an ellipse was plotted representing the locus of colour differences from the centre. The ellipse is effectively a cross-section of the three-dimensional ellipsoid around the same colour centre. Such ellipsoids are defined by the lengths of the semi-axes of symmetry: lightness, metric chroma and hue, hence the ellipse is defined by the lengths of the semi-axes of just metric chroma and hue.

Investigations by Strocka et $a l$. [6] and Luo and Rigg [7] illustrated how the comparison of sets of ellipses can be complicated by many factors. These include physical and experimental aspects such as the use of different substrates, different levels of luminance, different numbers of observers and samples, reference differences and use of different scales. Luo and Rigg devised a method of putting such ellipses on a common scale in order to compare data from various sources. They studied a total of 188 colour centres, from which they obtained a set of 132 reliable ellipses and only +5 reliable ellipsoids. The convenience of generating difference pairs digitally offers the prospect of detailed and comprehensive coverage.

\section{The Shademaster}

The Shademaster is a computer-aided colour specification, manipulation and communication system that is capable of displaving on screen a total or 16 million colours defined by CIE courdinates [11-12]. tpur: $\pi$ rom the main processing unit, the svetem ulato onsist is a Minovita colour TV analyser, a $51 \mathrm{~cm}$ colour monitor, a lighting cabinet for viewing physical coloured samples, a keyboard and a mouse which enables the observer to move to whichever area of the screen he wishes to work in. The screen is designed to show a graphic representation of the inside of a lighting cabinet or viewing box identical to those used in the coloration industry as a standard piece of equipment. Extensive experiments have shown that a colour displayed on the Shademaster screen is a close match to a physical sample with the same coordinates. The visual context of the screen is important. For example, Hunt has shown that there are significant appearance differences when isolated screen colours in a dark surround are compared with physical samples [13].

The system can communicate colours using any of four different sets of coordinates: $X Y Z, x y Y, L a b$ and $H C L$, as well as having the capacity to alter the RGB values of the colour under examination. If necessary, it is then possible to formulate a recipe for a dye, ink or paint to match the displayed colour. The CIELAB and CMC(1:1) perceptibility formulae are both programmed into the computer which enables the observer to obtain $\Delta E, \Delta H, \Delta C$ and $\Delta L$ values at the touch of a button. With Shademaster it is possible to increase or decrease the value in one or more of the three dimensions of hue, chroma or lightness independently. This, and the ability of the system to display several million different colours, means that the observer can view an infinite number of colour-difference pairs.

\section{OBJECTIVES}

A major unsolved problem for the coloration industries is the development of an accurate relationship between visual colour difference perception and colour measurement values. Although the $\mathrm{CMC}(l: c)$ equation has been established in a British Standard, there is still a need to gather further visual data in order to test the equation. The primary objective of this study was to explore the use of on-screen colour to assess the performance of the $\mathrm{CMC}(l: c)$ formula as a measure of visible colour difference. In order to draw worthwhile conclusions, the experiment devised would have to incorporate a series of colour centres reasonably distributed throughout the spectrum as well as various measurements taken in each of the hue, chroma and lightness directions.

The Shademaster allows an observer to detect a colour difference at its threshold of visibility. Hence the user can ascertain how easily a small colour difference can be detected on the screen. The resultant data from such observations can be used to answer three further questions.

(a) How does the visibility vary from colour to colour? For instance, it may be easier to detect a colour difference at a blue colour centre than at a green or a red centre

(b) How does the visibility vary with direction? Is the colsur difference easier or more difficult to detect in one direction than in the other two? For example, a colour difference in the plus or minus hue direction of 
one colour centre may be easier to see than in the chroma or lightness direction of that same colour centre.

(c) Can boundaries of visibility be reliably established around each colour centre?

\section{EXPERMMENTAL DESIGN}

Colour-difference measurement has proved to be a multivariable problem. Because this set of experiments uses sample pairs displayed on a computer screen, as opposed to dyed or painted samples, variables related to the physical properties of coloured samples, such as the use of different substrates or surface textural effects, can be eliminated at the beginning. Relevant variables include those relating to the colour under examination, the system, the display and observation. It is therefore necessary to eliminate variables until a single set can be isolated for examination.

Before each set of measurements was recorded, the Shademaster system was switched on and left for a short while until complete calibration had taken place, keeping all system variables constant.

The measurements were taken for a range of nine different colour centres covering all areas of the spectrum. Table 1 gives details for each colour. Hue angle $\theta$ can be calculated using Eqn 2:

$$
\tan \theta=b / a
$$

a hue angle of $0^{\circ}$ being the red axis of $L a b$ space, $90^{\circ}$ being the yellow axis, $180^{\circ}$ the green axis and $270^{\circ}$ the blue axis.

It is unlikely that a threshold of visibility can be determined accurately from a single observation. Therefore, for this investigation, a total of ten sets of measurements were recorded by a group of observers.

The accuracy of judgements made at the threshold of visibility between the colours depends on the method used for the experiment. The three primary methods of judgement were stated by Hill as being [8]:

(a) Method of adjustment or average error

(b) Method of limits

(c) Method of constant stimuli.

Table 1 Nine colour centres

\begin{tabular}{llllrr}
\hline Colour & $\begin{array}{l}\text { Hue } \\
\text { angle (deg) }\end{array}$ & $c$ & $L$ & $a$ & \multicolumn{1}{c}{$b$} \\
\hline Blue & 284.01 & 40.27 & 29.41 & 9.75 & -39.07 \\
Purple & 314.19 & 35.36 & 42.90 & 24.65 & -25.35 \\
Pale blue & 248.90 & 21.32 & 67.47 & -7.68 & -19.89 \\
Green & 165.89 & 32.11 & 60.40 & -31.14 & 7.83 \\
Yellow & 82.62 & 50.69 & 81.78 & 6.51 & 50.27 \\
Orange & 48.58 & 47.18 & 65.39 & 31.21 & 35.38 \\
Pink & 325.25 & 28.15 & 66.78 & 23.13 & -16.05 \\
Red & 35.66 & 69.49 & 52.61 & 56.46 & 40.51 \\
Brown & 32.26 & 39.59 & 44.38 & 33.48 & 21.13 \\
& & & & & \\
\hline
\end{tabular}

In this case, the third method was employed, the definition of which was given as follows: 'The method of constant stimuli uses many trials on each of which the magnitude of the stimulus property is fixed (i.e. constant). The subject is required to make either an absolute or a comparative judgement on each trial, the essential feature being that the stimulus properties are not varied during an observation. The threshold is determined statistically in all trials in which the stimulus is presented several times at each of many discrete magnitudes' [8]. For this investigation a pair of colours with a fixed difference between them was presented one at a time, and exactly the same set of differences was shown to each of the ten observers.

The Shademaster screen represents a perspective view of the inside of a lighting cabinet, showing a grey background with grey and coloured squares representing a central tile and various reference tiles. For this investigation the central tile was used to compare the standard and sample colours, which was achieved by dividing the tile into two equal halves. The right-hand half represented the standard, whose coordinates were equal to those of one of the nine colour centres. The left-hand half of the tile was the sample, whose colour was to be altered. The two halves of the tile were lying adjacent to one another with neither a gap nor a visible dividing line between them. There were no edge clues present, which means the edges of the two halves were perfectly aligned all the way around, i.e. there was no staggered or overhanging effect and so when both halves were of the same colour, the central tile did not appear to be divided.

\section{Quantisation effect}

When a colour difference was being created between the two tiles, it was found that it did not increase or decrease in a continuous, uniform manner, but in discrete steps relating to the integer change in the values of $R, G$ and $B$ on a scale of 0 to 255. To determine the points at which these steps occurred, the value of hue, chroma or lightness was altered until there was a change in one or more of the $R, G$ or $B$ values. These values were recorded for every individual step, hence allowing each one to be quickly and easily reproduced. This process was repeated until a series of pure hue, chroma or lightness steps of increasing magnitude had been defined for each direction and dimension over the nine colour centres. The $\Delta E, \Delta H, \Delta C$ and $\Delta L$ values were measured for each step and the data recorded for future processing. By this process it was possible to derive sequences of $R, G$ and $B$ changes close to the desired vector, e.g. pure hue or chroma change. The step sizes in terms of $\Delta E$ were relatively coarse. Detailed mapping of visibility boundaries may require $R G B$ definition on a 0 to 1024 scale, with much reduced step size. This level of quantisation has been achieved experimentally on Shademaster, but has not yet been validated for use in colour-difference work. For this work accurate boundaries were determined by an indirect approach. 


\section{Experimental procedure}

Using the mouse, the sample colour was changed in steps by increasing or decreasing its hue, chroma or lightness. In the tests for this experiment only one dimension was altered at a time, the remaining two being kept constant. For example, when the colour was varied in the chroma direction only, the values of hue and lightness remained unchanged. This ensured that the differences generated in each dimension were as pure as possible.

The colour was altered one step at a time in order to determine at which point the difference between the standard and the sample was just noticeable to the observer A 'blink' function, which allows the observer to blink the sample half of the tile back to the same colour as the standard, was provided.

All the observers were asked to examine each colour pair. They were asked the question: 'Is the colour difference visible? . They wrote their answer $\Omega$ for yes, $N$ for no) in a table provided for them. Results were recorded in the positive and negative directions of hue, chroma and lightness, generating one set of measurements in each direction across the nine colour centres.

\section{Measurement of colour difference pairs}

Each colour-difference pair was measured in turn using a Bentham telespectroradiometer (TSR). In accordance with the manufacturer's specifications, the system was recalibrated against a tungsten lamp before recording the measurements in order to achieve a greater accuracy of results. The apparatus was arranged in such a way that the telescope was exactly $1 \mathrm{~m}$ away from the colour monitor and pointing directly at the centre of the screen. This position was maintained throughout the whole series of measurements so that every colour difference was measured under exactly the same conditions. For each pair the standard colour was first brought up on the screen and measured, then the sample colour was brought up immediately afterwards and measured. The whole process was carried out in a darkened room in order to minimise the effects of ambient light. The resultant $\Delta E, \Delta H$, $\Delta C$ and $\Delta L$ values of each colour-difference pair could then be calculated.

The Shademaster calibration system has been shown to reproduce CIE colour specifications accurately [10-12]. However, continuously variable CIE coordinates are mapped onto discrete integer $R G B$ values. The Bentham results were therefore validated by using the reverse transformation of RGB to CIE coordinates. Given two sets of $R G B$ values, the monitor calibration system was used to calculate $\Delta x, \Delta y$ and $\Delta Y$ exactly for a particular set of $\Delta R, \Delta G$ and $\Delta B$, therefore providing a direct cross-check on the Bentham TSR measurements. A set of reverse mapped $\Delta E$, $\Delta H, \Delta C$ and $\Delta L$ values was recorded for each colourdifference pair and compared with those obtained from the Bentham.

\section{Statistical analysis}

Methods of calculating correlation are used to determine the way in which values vary with respect to one another between various sets of data. If values increase or decrease together, they are said to be positively correlated. Conversely, if two sets vary inversely, i.e. one increases as the other decreases or vice versa, then they are said to be negatively correlated. Perfect positive correlation is given a value of +1 whereas perfect negative correlation is given a value of -1 . Two sets of data having no correlation would be given a value of zero.

The oldest and most widely used measure of correlation is known as the Pearson product moment correlation coefficient and it is symbolised by the letter $r$, calculated using Eqqn 3:

$$
r=\frac{n \Sigma x y-(\Sigma x)(\Sigma y)}{\sqrt{\left[n \Sigma x^{2}-(\Sigma x)^{2}\right]\left[n \Sigma y^{2}-(\Sigma y)^{2}\right]}}
$$

where $x$ and $y$ represent the data in the two sets and $n$ is the number of pairs of values

Eqn 3 can be used as a means of testing hypotheses, the null hypothesis being that the true population correlation is equal to zero. The alternative hypothesis states that the true population correlation is not zero and therefore a valid relationship exists between the two sets of data. If the value of $r$ is too low, then the null hypothesis cannot be rejected. The significance level of the test can be obtained from a table of critical minimal values of $r$.

To to compare the results obtained from the Bentham TSR and those calculated using the reverse mapping method, their correlation coefficients were calculated using Eqn 3, taking the Bentham results as the $X$ values and the calculated results as the $Y$ values (Table 3 ).

\section{RESULTS}

The results from the visual judgements of perceptibility can be seen in Table 2. For each colour at each step, Table 2 shows a pair of numbers corresponding to the decisions of the ten observers. The first figure of each pair represents the number of observers to whom a colour difference was visible and the second figure represents the number of observers to whom the difference was not visible. For example, for the second step in the $+C$ direction at the purple colour centre, a colour difference was visible to seven observers, whereas three observers could not see a difference.

This data was used to plot graphs of perceptibility at each step for plus and minus hue, chroma and lightness differences. To obtain a numerical value for perceptibility, each 'yes' decision was given a value of +1 and each 'no' decision a value of -1 . The sum of all the numbers relating to the judgements provides a value of perceptibility between +10 (when the difference was visible to all observers) and -10 (when the difference was not visibie to any of the observers). 
Table 2 Perceptibility data

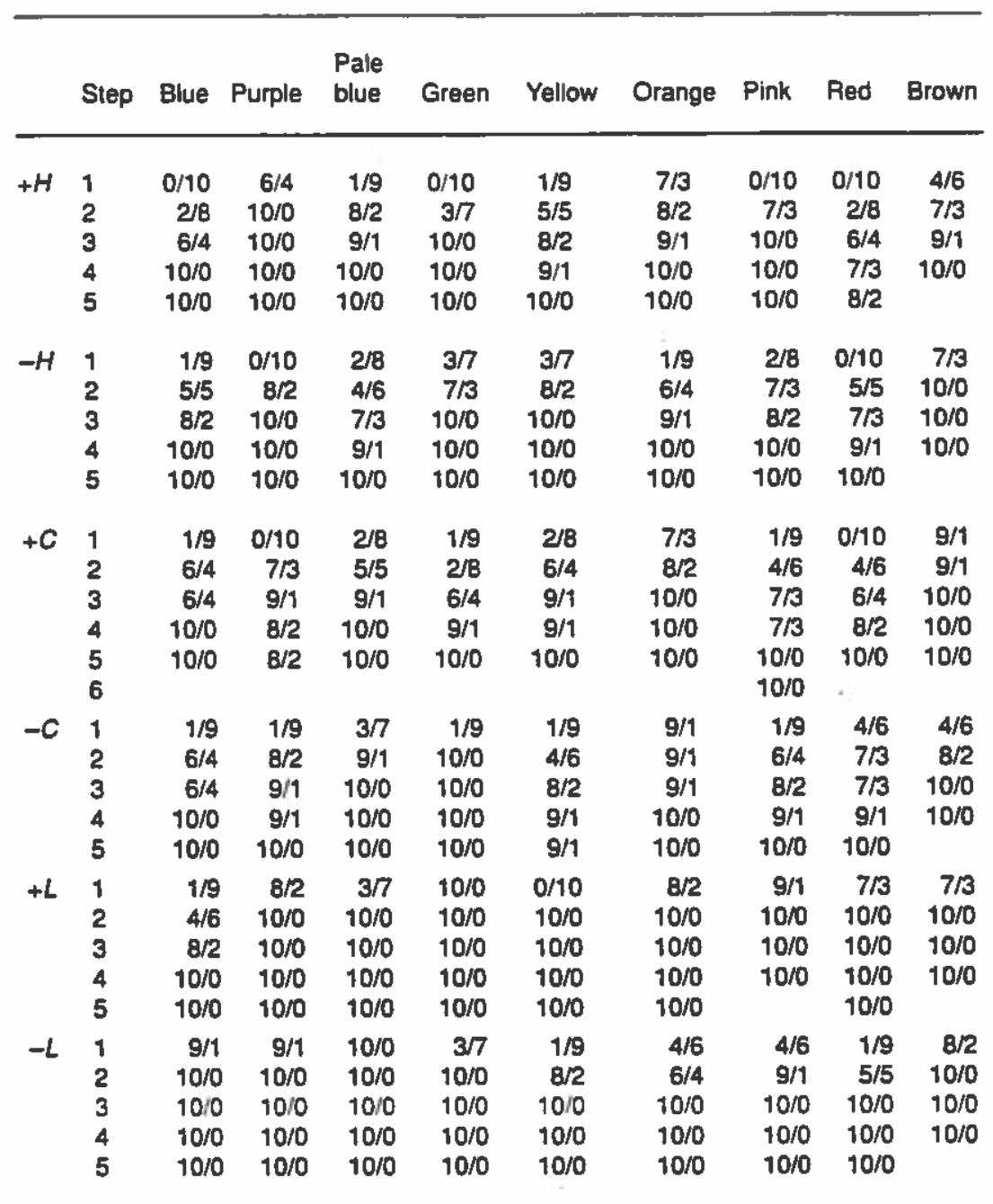

It would be impractical to include the full set of 27 graphs here, but as an example those for the blue colour centre can be seen in Figure 2(a-c). Each graph shows two curves, one being the perceptibility of the colour difference in the plus direction and the other in the minus direction.

\section{$H / C$ diagrams}

The calculated values of $\Delta H$ and $\Delta C$ were plotted for the hue and chroma changes in $H / C$ diagrams; sample plots are given in Figure $3(a-c)$. These diagrams represent a small area of $\mathrm{CMC}(1: 1)$ colour space around the colour centre. In this colour space the tolerance ellipsoid becomes a sphere of radius $\Delta E=1$. Three different symbols are used to show the distribution of all the points: one denoting a 'visible' majority decision, another denoting a 'not visible' majority decision and a third for those points at which there was an equal number of both decisions. Using graphs of perceptibility such as those shown in Figure 2, it is possible to estimate, by interpolation, the approximate position of the visibility boundary in each direction. Again taking the blue centre as an example, from Table 2 we can see that the colour difference in the plus hue direction was only visible to two out of the ten observers at step two, but was visible to six of the ten observers at step three. This clearly suggests that the perceptibility boundary lies somewhere between steps two and three. Referring to Figure 2(a), we can see that the boundary is approximately three-quarters of the way between steps two and three. This can therefore be plotted between the points on the $H / C$ diagram corresponding to step two and step three in the plus hue direction (Figure $3(a))$. With the exception of the orange centre, this process was repeated for each colour difference in the plus and minus hue and chroma directions, producing a locus of points around each colour centre that can be considered to be an approximate boundary of perceptibility. The $C M C(1: 1) \triangle E$ unit ellipses were also plotted on the same set of axes in order to compare the two.

Table 2 and Figure 3(c) show that with the orange colour centre all but one of the colour differences were visible at the first step, and so there is insufficient information for estimating a perceptibility boundary by the method used for the other eight colours. Apart from the minus hue direction, where the visibility limit lies between steps one and two, it must be assumed that the 

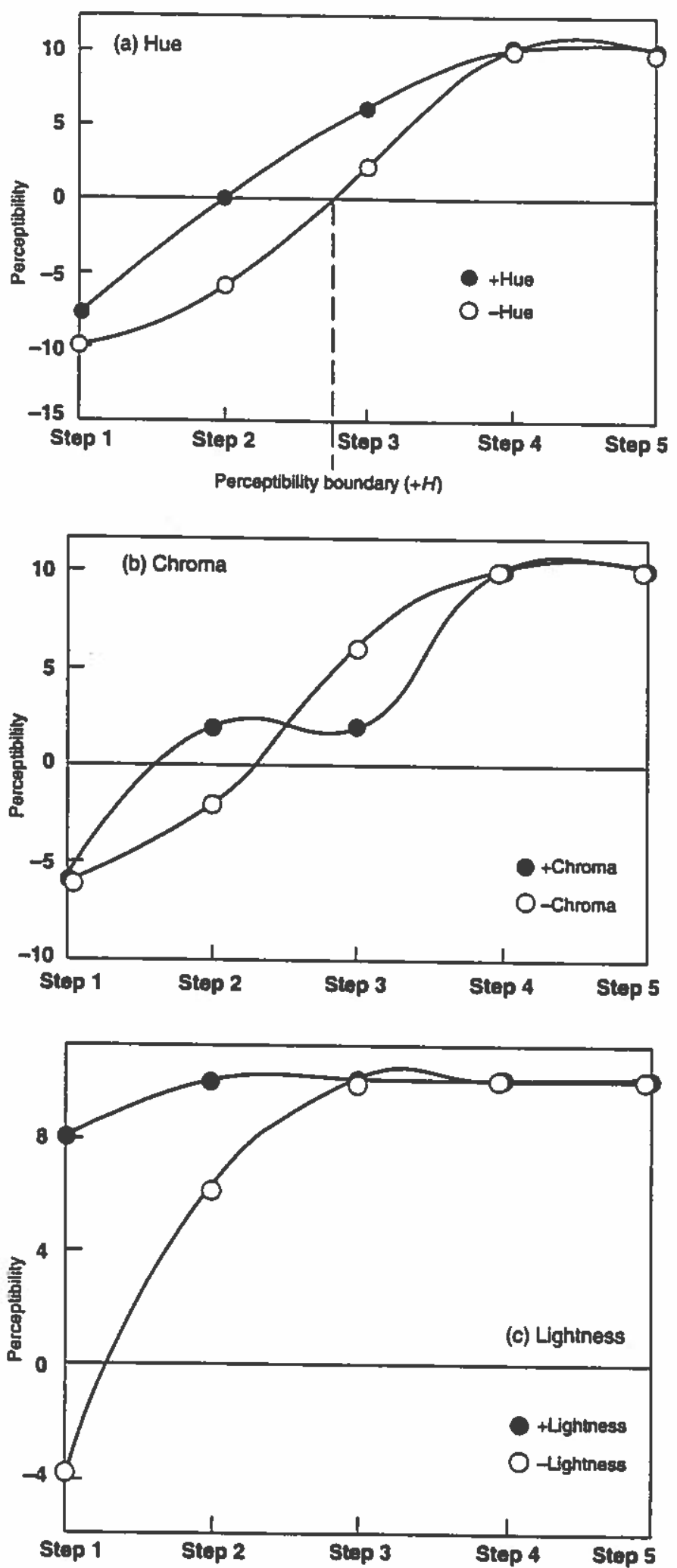

Figure 2 Perceptibility curves for colour differences (blue centre)

boundary of visibility for the orange centre lies somewhere inside those points on the graph corresponding to the first steps in the plus hue and plus and minus chroma directions.

The full tabulated values of $\Delta E, \Delta H, \Delta C$ and $\Delta L$ for both the Bentham and calculated results were too numerous to include in this report, but all are available from the
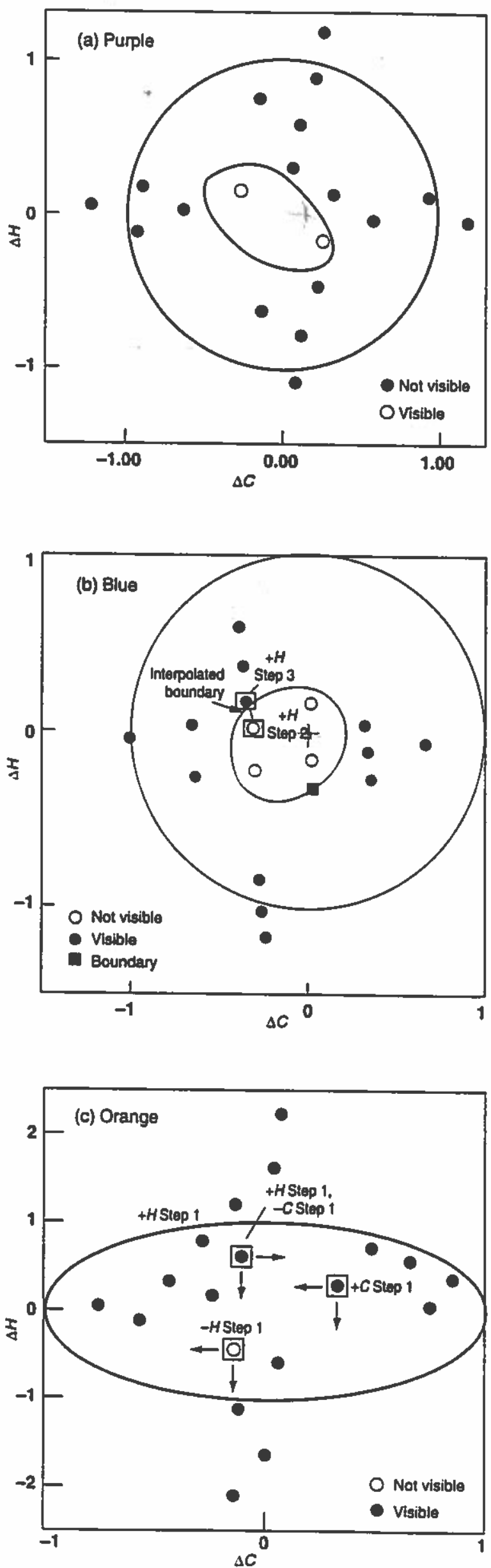

Figure 3 Estimated boundaries of perceptibility for three colour centres 


\section{CONCLUSIONS}

The Shademaster system has been used to investigate the reliability of the $\mathrm{CMC}(l: c)$ formula as a measurement of small colour differences. After measuring each of the colour-difference pairs used by two separate methods, and having proved that there is a high positive correlation between the two resultant sets of data, it can be assumed that a reasonably accurate set of quantised steps has been established at a confidence level of at least $95 \%$. It follows that interpolated perceptibility boundaries can be defined around each colour centre to within the same confidence limits.

Further validation work is in progress to relate judgement of physical samples to judgement of identical differences on-screen. The stepwise nature of colour change on-screen would be expected to cause no problems given that 16.7 million colours are available. In practice, unit change in $R, G$ or $B$ produces $\Delta E$ changes as large as 0.6 in some cases. Experiments are in hand to increase colour choice from $256^{3}$ to $1024^{3}$ possible colours. This reduces the worst case $\Delta E$ step size to about 0.15 .

The anomalous relationship between $\Delta E$ and visibility found in the lightness results throw some doubt on the validity of treating colour difference as a threedimensional Euclidian measure. A preliminary analysis will be published separately and further experimentation pursued.

From the information gained by statistical analysis and the fact that each set of observations for all the colour centres in this study shows a logical progression of visibility judgements, it is possible to accurately produce and measure just-noticeable colour differences on a computer screen. This eliminates the need to produce dyed or painted physical samples. It can also be deduced that the $\mathrm{CMC}(1: 1)$ formula is a reasonable measure of colour difference, although further work is necessary in the area of computer-simulated small colour differences.

\section{REFERENCES}

1. KMcLaren, J.S.D.C., 86 (1970) 354.

2. KMCLaren, J.S.D.C., 92 (1976) 338.

3. F J J Clarke, R McDonald and B Rjgg J.S.D.C., 100 (1984) 128.

4. KMcLaren, Text. Chem. Colorist, 21 (1989) 33.

5. D L Macadam, J. Opt. Soc. Amer., 32 (1942) 247.

6. D Strocka, A Brockes and W Pafthausen, Col. Res. Appl., 8 (1983) 169.

7. M R Luo and B Rigg, Col. Res. Appl., 11 (1986) 25.

8. A Hill in Colour physics for industry, Ed. R McDonald (Bradford: SDC, 1987).

9. M R Luo and Rigg, J.S.D.C., 103 (1987) 86.

10. D P Oulton and I Forat, I. Tetile Inst. 83 (3) (1992).

11. D P Oulton adn C J Hawkyard, Spazio Tess., 5 (June 1991) 65 (English translation).

12. CJ Hawkyard and D P Oulton, J.S.D.C. 101 (1991) 309.

13. R W G Hunt, Col. Res. Appl, 16 (1991) 146.

14. MR Luo and B Rigg, J.S.D.C., 103 (1987) 126.

15. RMCDonald, J.S.D.C., 96 (1980) 372

16. RMcDonald, J.S.D.C., $96(1980) 418$.

17. RMcDonald, Col. Res, AppL, 15 (1990) 249.

18. KWitt, Col. Res. Appl., 12 (1987) 128.

19. D H Alman et al., Col. Res. Appl., 14 (1989) 139.

20. R S Berns et al., Col. Res. Appl., 16 (1991) 297.

21. D B Judd and $G$ Wyszeck. Colour in business science and industry, 3rd Edn (New York: John Wiley, 1975).

22. G A Agoston, Colour theory and its applications in art and design (Berlin: Springer, 1979).

23. W D Wright, The measurement of colour (London: Adam Hilger, 1969).

24. I D Mollon and L T Sharpe, Colour vision: physiology and psychophysics, Proc. Cambridge Colour Vision conf 1982 (London: Academic Press, 1983).

25. J D Wynne, Learning statistics - a common sense approach (London: Macmillan, 1982).

26. J Cohen and P Cohen, Applied multiple regnession/correlation for the behavioural sciences (New Jersey: Lawrence Earlbaum Assocn, 1975). 
authors. However, the correlation coefficients between the two sets of results are shown in Table 3. From this we can see that in general there was a very high correlation between the Bentham and the calculated values of $\Delta E, \Delta H$, $\Delta C$ and $\Delta L$

\section{DISCUSSION}

It is clear from the summary of results in Table 2 that, for the majority of the nine colour centres, the ten observers were in good agreement on when a difference was visible. A lightness difference was visible at the second step and some differences (green $+L$ and pale blue $-L$ ) were even visible to all observers at the first step. However, for the changes in hue and chroma, the differences were visible to the majority of observers only at the third or fourth step. While precise boundaries for lightness difference visibility could not be deduced from our measurements, it is clear that observers agreed that they lay on average at a $\Delta E$ value of less than 0.34 . There was also clear evidence of anomalous behaviour between $\Delta E$ and the visibility of lightness differences, which will be reported elsewhere.

It is possible to draw comparisons from the perceptibility diagrams shown in Figure $3(a-c)$. In each case the outer curve represents a tolerance level of $\Delta E=1$, and the inner curve represents the approximate estimated boundary of perceptibility for that colour centre. Because of the lack of points for invisible differences, the position of the perceptibility boundary around the orange colour centre can only be assumed to lie within the points indicated in Figure 3(c), which correspond to the first steps in the plus hue and plus and minus chroma directions.

Observers were unanimous that they could see much smaller hue differences than that indicated by $\Delta E$ equal to one at all colour centres. Figure 3 gives estimated boundaries for hue difference visibility in the $\Delta E$ range of $0.25-0.8$, depending on colour. This lends weight to the common statement that hue differences are less desirable than chroma differences. The observers also agreed that, as measured by $\mathrm{CMC}(1: 1), \triangle E$ chroma differences were less visible than hue differences. A rough ratio of nearly 2:I was visible in these preliminary investigations at some colour centres (Figure 3). They were also unanimous that they could see chroma differences significantly less than a $\triangle E$ value of one in chroma.

In the set of results on pairs differing in lightness, observers produced anomalous correlation between visibility and $\Delta E$. These will be analysed and reported separately. They were, however, all in agreement that they could see lightness differences of $C M C(1: 1) \triangle E$ of less than 0.6 at all colour centres, and a minimum $\Delta E$ of 0.18 at the red centre.

Table 3 generally shows excellent correlation between the Bentham and calculated results for $\Delta E, \Delta H, \Delta C$ and $\Delta L$, many values representing near perfect positive correlation. On average, the correlation is high with an overall significant level of $95 \%$. The null hypothesis that states there is no correlation between the two sets of data can therefore be rejected.

Table 3 Correlation coetficients between Bentham and calculated results

\begin{tabular}{|c|c|c|c|c|c|c|c|c|c|c|}
\hline & Step & Blue & Purple & $\begin{array}{l}\text { Pale } \\
\text { blue }\end{array}$ & Green & Yellow & Orange & Pink & Red & Brown \\
\hline$\Delta \mathbf{E}$ & $\begin{array}{l}+H \\
-H \\
+C \\
-C \\
+L \\
-L\end{array}$ & $\begin{array}{l}0.99 \\
0.99 \\
0.99 \\
0.99 \\
0.74 \\
0.76\end{array}$ & $\begin{array}{l}0.99 \\
0.99 \\
0.99 \\
0.99 \\
0.89 \\
0.91\end{array}$ & $\begin{array}{l}0.99 \\
0.99 \\
0.99 \\
0.96 \\
0.85 \\
0.93\end{array}$ & $\begin{array}{l}0.99 \\
0.99 \\
0.99 \\
0.99 \\
0.93 \\
0.56\end{array}$ & $\begin{array}{l}0.99 \\
0.66 \\
0.99 \\
0.99 \\
0.83 \\
0.84\end{array}$ & $\begin{array}{l}0.99 \\
0.99 \\
0.93 \\
0.97 \\
0.95 \\
0.89\end{array}$ & $\begin{array}{l}0.61 \\
0.99 \\
0.99 \\
0.99 \\
0.61 \\
0.72\end{array}$ & $\begin{array}{l}0.99 \\
0.99 \\
0.98 \\
0.98 \\
0.98 \\
0.88\end{array}$ & $\begin{array}{l}0.99 \\
0.99 \\
0.81 \\
0.99 \\
0.99 \\
0.93\end{array}$ \\
\hline$\Delta H$ & $\begin{array}{l}+H \\
-H \\
+C \\
-C \\
+L \\
-L\end{array}$ & $\begin{array}{l}0.99 \\
0.99 \\
0.63 \\
0.99 \\
0.92 \\
0.95\end{array}$ & $\begin{array}{l}0.99 \\
0.99 \\
0.97 \\
0.38 \\
0.96 \\
0.97\end{array}$ & $\begin{array}{l}0.99 \\
0.99 \\
0.93 \\
0.96 \\
0.95 \\
0.82\end{array}$ & $\begin{array}{l}0.99 \\
0.99 \\
0.92 \\
0.94 \\
0.78 \\
0.32\end{array}$ & $\begin{array}{l}0.99 \\
0.51 \\
0.49 \\
0.97 \\
0.84 \\
0.86\end{array}$ & $\begin{array}{l}0.99 \\
0.99 \\
0.99 \\
0.98 \\
0.98 \\
0.22\end{array}$ & $\begin{array}{l}0.72 \\
0.99 \\
0.75 \\
0.99 \\
0.99 \\
0.99\end{array}$ & $\begin{array}{l}0.99 \\
0.92 \\
0.99 \\
0.54 \\
0.88 \\
0.99\end{array}$ & $\begin{array}{l}0.99 \\
0.99 \\
0.99 \\
0.99 \\
0.77 \\
0.52\end{array}$ \\
\hline$\Delta C$ & $\begin{array}{l}+H \\
-H \\
+C \\
-C \\
+L \\
-L\end{array}$ & $\begin{array}{l}0.99 \\
0.97 \\
0.98 \\
0.99 \\
0.86 \\
0.97\end{array}$ & $\begin{array}{l}0.96 \\
0.87 \\
0.99 \\
0.99 \\
0.97 \\
0.91\end{array}$ & $\begin{array}{l}0.99 \\
0.98 \\
0.99 \\
0.99 \\
0.86 \\
0.75\end{array}$ & $\begin{array}{l}0.35 \\
0.98 \\
0.99 \\
0.99 \\
0.78 \\
0.64\end{array}$ & $\begin{array}{l}0.94 \\
0.01 \\
0.99 \\
0.99 \\
0.50 \\
0.89\end{array}$ & $\begin{array}{l}0.48 \\
0.93 \\
0.99 \\
0.99 \\
0.36 \\
0.55\end{array}$ & $\begin{array}{l}0.06 \\
0.96 \\
0.99 \\
0.99 \\
0.96 \\
0.98\end{array}$ & $\begin{array}{l}0.94 \\
0.15 \\
0.99 \\
0.98 \\
0.09 \\
0.77\end{array}$ & $\begin{array}{l}0.90 \\
0.76 \\
0.83 \\
0.99 \\
0.18 \\
0.43\end{array}$ \\
\hline$\Delta L$ & $\begin{array}{l}+H \\
-H \\
+C \\
-C \\
+L \\
-L\end{array}$ & $\begin{array}{l}0.98 \\
0.69 \\
0.96 \\
0.82 \\
0.99 \\
0.99\end{array}$ & $\begin{array}{l}0.99 \\
0.98 \\
0.96 \\
0.93 \\
0.99 \\
0.99\end{array}$ & $\begin{array}{l}0.89 \\
0.99 \\
0.97 \\
0.87 \\
0.99 \\
0.99\end{array}$ & $\begin{array}{l}0.94 \\
0.79 \\
0.57 \\
0.97 \\
0.99 \\
0.79\end{array}$ & $\begin{array}{l}0.91 \\
0.85 \\
0.36 \\
0.27 \\
0.97 \\
0.99\end{array}$ & $\begin{array}{l}0.96 \\
0.98 \\
0.99 \\
0.94 \\
0.99 \\
0.90\end{array}$ & $\begin{array}{l}0.97 \\
0.98 \\
0.08 \\
0.79 \\
0.99 \\
0.99\end{array}$ & $\begin{array}{l}0.59 \\
0.65 \\
0.69 \\
0.06 \\
0.99 \\
0.99\end{array}$ & $\begin{array}{l}0.99 \\
0.89 \\
0.56 \\
0.97 \\
0.99 \\
0.91\end{array}$ \\
\hline
\end{tabular}

